I have used the phrases lines of inductive force and curved lines of force in a general sense only, just as we speak of lines of magnetic force.

$\mathrm{He}$ does not represent field intensity by lines.

Maxwell, however, changed the meaning by calling Faraday's lines of force lines of induction and using the term lines of force for lines of intensity only.

And we? We use the words sometimes in Faraday's sense, sometimes in Maxwell's sense. We introduce them when speaking of field intensity and later on make the glaring mistake of asserting that the induced electromotive force is measured by the cutting of lines of force. The American Institute of Electrical Engineers has proposed to call the unit of magnetic intensity the "gauss"; it seems to be a general understanding, judging from papers appearing on magnetic subjects, that it is also the unit of induction. Personally I prefer to discard the troublesome term altogether, but it may be that it has become so familiar to the scientist and is so generally used in engineering practise, though usually there in the meaning of lines of induction, that it is too late to abolish it altogether. If we must keep the lines of force in our text-books, let us use them in one sense only. We should certainly stop confusing our students about the real nature of these two totally different quantities. $^{3}$

I hope to have proven that we lack in the presentation of several topics that accuracy of expression of which in general the physicist can be justly so proud, and that greater uniformity in the use of certain terms is very desirable. Our ideas as to the fitness of proposed names for the quantities in question as well as to the choice of definitions, may be widely different. Your speaker clearly realizes that

${ }^{3}$ See also a paper by Professor Patterson, "Michigan Technic," 20, No. 2, p. 35, 1907. there is ample room for discussion and that the sporadic attempt of a single scientist to correct the apparent faults in our teaching can not better the conditions appreciably.

Reforms of a lasting nature can be accomplished and the desired result reached in shortest time, only, if definite propositions be made by a committee consisting of a number of representative physicists. With their influence behind a reform movement of this kind we shall soon reach practical unanimity.

In conclusion, let me assure you from my own experience that it is not an extremely difficult matter to teach the student to make these fine distinctions between different physical quantities. It is true, it requires some deep and accurate thinking; but the result has always been that in the end the subject has become clearer to the student and, as I have been assured, even more interesting.

K. E. Guthe

\section{THE EVOLUTION OF INTELLIGENCE AND ITS ORGANS ${ }^{1}$}

WE recognize two very distinct types of physiological functions: (1) activities concerned with the inner working of the bodily mechanism-nutrition, internal regulation, etc. - and called vegetative or visceral functions; (2) activities concerned with the adjustments of the body to outside, or environmental influences. These we call somatic functions.

These reaction types are, of course, always intimately related and interdependent; nevertheless, as we ascend the scale of animal life the history of the evolution of both structure and function shows a progressive elaboration of each of these

${ }^{1}$.Address of the vice-president and chairman of Section F-Zoology. American Association for the Advancement of Science, Boston, 1909. 
two functional systems and differentiation from the other, so that in higher vertebrates the distinction between them may be said to be fundamental both to anatomy and to physiology.

As children we probably considered the chief distinction between plants and animals to be the ability of the latter to move freely about; but one of the first lessons in our elementary biology was the correction of this notion by the study of sedentary animals and locomotor plants. Nevertheless, I fancy that in the broad view the childish idea has the root of the matter in it. The plants and sedentary animals may have their vegetative functions of internal adjustment never so highly specialized and yet remain relatively low in the biological scale because their relations with the environment are necessarily limited to the small circle within which they first take root, whereas the power of locomotion carries with it, at least potentially, the ability to choose between many more environmental factors. It is only the free-moving animals that have anything to gain by looking ahead in the world, and here only do we find well-developed distance receptors, $i$. e., sense organs adapted to receive impressions from objects remote from contact with the body. And the distance receptors, as we shall see, have dominated and set the direction of the evolution of the nervous system in vertebrates.

Thus arose the animal head, with its three important functions of feeding, breathing and the recognition of mates and enemies. Parker has recently reviewed ${ }^{2}$ in an illuminating way the earlier stages in the differentiation of the nervous system and I shall not attempt to go over this ground again, but will take a bilaterally symmetrical segmented animal with a differentiated head end as the point of de-

\footnotetext{
2 Parker, G. H., Popular Science Monthly, 1909.
}

parture in an examination of the phylogenetic history of behavior types.

On anatomical and zoological grounds zoologists are in the habit of subdividing the animal kingdom in the way roughly suggested by the accompanying scheme. Most of the important groups are naturally arranged in two great phyla which have apparently been quite distinct as far down as the flat worms. One of these, which we may call the articulate phylum, includes

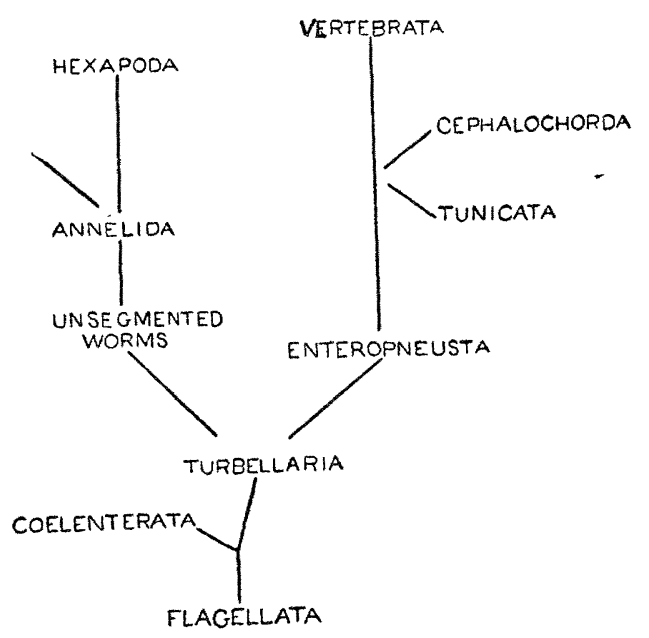

the segmented worms, crustaceans, spiders and insects; the other phylum, after passing through a series of obscure invertebrate stages, largely at the present time extinct, culminates in the true vertebrates. It may be termed briefly the vertebrate phylum, and all of its members, from the lowest to the highest, are sharply distinguished from those of the articulate phylum by several characteristics, among which is the development of mesodermal gut pouches. All forms above the Enteropneusta have gill clefts, either embryonic or adult, which likewise develop as gut pouches and a dorsal tubular nervous system, which is derived from the mid-dorsal ectoderm and is separated from the gut by a supporting notochord. The articulates, on the other 
hand, have a ladder-like nervous system ventrally of the gut and of totally different origin.

These are illustrations of the nature of the data on the basis of which zoologists are in quite general agreement in recognizing the wide divergence of these two great phyla of metazoa.

Now, students of animal behavior have recognized also two fundamental behavior types among the higher animals. This is clearly stated by Yerkes when he points out $^{3}$ that the animal kingdom presents divergent lines in the development of action types.

Certain animals are markedly plastic or voluntary in their behavior, others are as markedly fixed or instinctive. In the primates plasticity has reached its highest known stage of development; in the insects fixity has triumphed, instinctive action is predominant. The ant has apparently sacrificed adaptability to the development of ability to react quickly, accurately and uniformly in a certain way. Roughly, animals might be separated into two classes: those which are in high degree capable of immediate adaptation to their conditions, and those which are apparently automatic since they depend upon instinctive tendencies to action instead of upon rapid adaptation.

If time permitted us to develop this conception, many striking illustrations might be cited of the predominance of now one, now the other, of these action types in different animals. The most striking feature of such an examination is the discovery that, while the generalized members of both of the zoologists' phyla exhibit an extreme development of neither action type, those forms which are structurally highly specialized generally have one or the other action type also highly developed; and in these cases an arrangement of animals according to their type of behavior follows closely the diphyletic ar-

${ }^{3}$ Journal of Comparative Neurology and Psychology, Vol. 15, 1905, p. 137. rangement previously elaborated on purely structural grounds. The anatomical basis of this harmony is readily apparent when the nervous systems of the two phyla are compared.

The central nervous system of the articulates is fundamentally a segmented ladderlike chain of nerve tissue with special ganglionic enlargements in the head related to the leading sense organs. It is dominated by the general body metamerism and the segmental reflex arcs are kept relatively distinct by the anatomical configuration. Some of the compound and chain reflexes are very complex; yet they tend to follow the appropriate stimuli with a mechanical precision which is simply an expression of the accurate working of a pre-formed mechanism. Since this is an inherited mechanism, all members of the species exhibit similar reactions and these do not require experience for their performance. This is instinct.

On the other hand, the vertebrate nervous system is fundamentally an epithelial tube, only imperfectly segmented, which contains not only direct reflex mechanisms of the articulate type, but also a massive continuous column of nerve cells and connecting fibers, the reticular formation, which is a diffuse correlation center related to all of the reflex arcs. In the head there are special enlargements derived from this (incompletely) segmented reticular formation, which make up the greater part of the brain in a higher vertebrate. These are the special correlation centers or the suprasegmental apparatuses of Adolf Meyer's terminology.

The entire vertebrate plan of nervous system is totally different from that of any member of the articulate series and, while adapted to perform stereotyped reflexes and instinctive modes of behavior, is also capable of wholly different types of reac- 
tion based on the functional plasticity of the reticular formation and its derivatives. There is, of course, some measure of plasticity in the behavior of arthropods, e. g., some ability to learn by experience, and they possess some tissue corresponding to the reticular formation; but in the broad view the distinctions just drawn are characteristic of the two phyla.

Without going into further detail, we may, then, generalize that the higher insects mark the culmination of the stereotyped or instinctive type of behavior, while the primates represent the culmination of the plastic, docile or rational type, and that the structural basis of this plasticity of the vertebrates is found in the relation of the reticular formation of their nervous systems to the other elements of the neural tube and especially in the suprasegmental correlation centers derived therefrom. The lower vertebrates are far inferior to the higher insects in many respects-often perhaps in the very ability to profit by experience of which we have been speaking; but their physical organization is such as to favor future differentiation in this direction, while that of the insects is such as to forbid it. Thus it appears probable that the dominance of the vertebrate type was foreshadowed far back among the ancestral crawling things in which no truly vertebrate character was manifest, foreshadowed merely by a structural type with different latent potencies.

The arthropod type of organization and action system is rigidly stereotyped in the race as well as in the individual; $i$. e., it tends to be transmitted without modification from generation to generation. Its pattern can be changed only by natural selection or some other agency which can act through heredity. The more plastic vertebrate type is not fixed completely at birth by heredity, but its precise form is more largely acquired as individual experience advances. As intelligence plays a progressively greater part in the behavior, infancy will be prolonged to afford the necessary opportunity for the plastic nervous system to be shaped in adaptation to the individual needs of the animal. The instruments of racial progress here are not merely natural selection acting through heredity, but also docility, social heredity and organic selection, acting largely through intelligent adaptation.

In the vertebrates the amount of preformed or inborn organization, both of structure and of function, is in general greater than in arthropods; but there is superposed upon this rigidly predetermined tissue in higher vertebrates the unspecialized embryonic correlation tissue, the details of whose organization are not laid down in the hereditary pattern, but are individually acquired during development. The ultimate pattern which will be assumed by this plastic tissue is largely shaped by the exigencies of function during the period of its immaturity and this in turn rests upon the nature of the environmental factors. In short, the educational period is limited to the age during which the epigenetic tissue, $i$. e., the correlation centers whose form is not predetermined in heredity, retains its plasticity under environmental influence.

Ultimately even the cerebral cortex matures and loses its power of reacting except in fixed modes. Its unspecialized tissue -originally a diffuse and equipotential nervous meshwork - becomes differentiated along definite lines and the fundamental pattern becomes more or less rigid. The docile period is past and, though the man may continue to improve in the technique of his performance, he can no longer do creative work. He is apt to say, "The dog is too old to learn new tricks." Whether 
this process occurs at the age of twenty or eighty years, it is the beginning of senility. And, alas, that this coagulation of the mental powers often takes place so early! Many a boy's brains are curdled and squeezed into traditional artificial molds before he leaves the grades at school. His education is complete and senile sclerosis of the mind has begun by the time he has learned his trade. For how many such disasters our brick-yard methods in the public schools are responsible is a question of lively interest.

We who seek to enter into the kingdom of knowledge and to continue to advance therein must not only become as little children, but we must learn to continue so. The problem of scientific pedagogy, then, is essentially this : to prolong the plasticity of childhood, or otherwise expressed, to reduce the interval between the first childhood and the second childhood to as small dimensions as possible.

The docile or educational period of a mammal is largely devoted to the progressive mechanization of the in-born plastic tissue of the higher correlation centers, $i . e$, to habit formation, or otherwise expressed to the elaboration of acquired automatisms and reflexes of the type commonly referred to as lapsed intelligence. Much confusion has arisen from the failure to distinguish these individually acquired automatisms from those performed in the hereditary pattern, $i$. e., lapsed intelligence from true instinct.

Now to return from this digression, let us consider some data bearing on the phylogeny of the nervous functions in vertebrates. We have commented upon the fact that the tubular form of the vertebrate nervous system presents mechanical advantages over the ladder-like form of the articulates for the development of correlation tissue and that the parent type of this tissue is found in the central gray and reticular formation which borders the gray matter in the spinal cord.

The nervous mechanism of the remarkable adaptiveness, the apparent purposefulness, of the spinal cord reflexes has been lucidly explained by. Sherrington in his Silliman lectures, where he shows that one of the chief functions of the correlation cells of the gray matter (cells of the reticular formation type) is the elaboration of a single final common path adapted to serve, as occasion may require, a large and variable number of receptors and afferent paths. Although this apparatus reacts largely in a fixed and invariable mode depending on the internal connections of the neurones of which it is composed, nevertheless it possesses a certain amount of flexibility growing out of a variable internal resistance at the synapses, or points of physiological union of one nerve cell with another, and particularly the modification of this resistance by practise or habit. This modifiability is not per se evidence of anything psychic; for we find it in unicellular animals and plants with no nervous system and even in many dead mechanisms; yet this feature is the point of departure for those higher types of correlation centers which serve as the organs of mind par excellence.

In the head end of the neural tube there is an obvious tendency for the peripheral nerves serving a single function to converge just before or just after they enter the brain so as to reach a single primary center. This concentration of functional systems is obviously advantageous in facilitating the distribution of afferent impulses to their proper motor organs, especially the total reactions so characteristic of vertebrate life as distinguished from the segmental reflexes characteristic of worms and insects. The enlargement of these primary 
sensory centers, which sometimes attain to enormous size, does not imply any more highly developed psychic powers than those of allied species with smaller brains; but rather a higher elaboration of certain reflex activities only.

The same is in large measure true of certain suprasegmental or secondary correlation centers. Thus, each one of the organs of higher sense discharges its afferent impulses into a massive primary receptive center and this in turn transmits it to correlation centers of the second, third and higher orders, where these nerve impulses are brought into relation with those from other sense organs and with the appropriate efferent pathways to the muscles or other organs of response. The correlation centers of this sort, which make up a large part of the thalamus and midbrain, are derivatives of the formatio reticularis tissue and are functionally of the same type. They permit of wonderfully complex discriminative reactions and are more readily modifiable by experience than are those of the spinal cord and medulla oblongata.

There is another type of highly developed correlation center whose psychic value is still less than the sensori-motor stations of which we have just been speaking. I refer to the central mechanism of what Sherrington calls the proprioceptive system. ${ }^{4}$ Of this the cerebellum is the most important example. The chief function of this system being the coordination and regulation of the skeletal musculature and other organs of somatic response (as distinguished from the interoceptive or visceral effectors), it is naturally purely reflex and its function is disturbed rather than facilitated by voluntary interference.

The correlation centers of the cerebral

"On the relation of this system to the exteroceptive, see my article on the "Morphological Subdivision of the Brain," Journal of Comparative Neurology and Psychology, Vol. 18, 1908, p. 395. hemispheres occupy a unique position. Their interpretation is possible only in the light of their origin in the lower groups of vertebrates. Numberless researches by our most able anatomists and physiologists have accumulated a vast wealth of data on this subject, which have, however, stubbornly resisted correlation and interpretation. Our debt to the generalizations and luminous terminology of Sherrington appears on almost every page of this address. Let us begin our inquiry into the origin of cortical function with an examination of a typical feeding reaction.

The primitive feeding reactions are very simple reflexes, but even in the lowest animals they are easily modifiable, as Jennings has shown for protozoa and Parker for sea anemones. Predaceous species among the lowly vertebrates commonly hesitate long before they strike, but once the action is initiated it follows to completion in a very precise and invariable fashion. The pike or the frog will watch the moving prey long before the forward leap is made to seize it; but when once taken it will generally be swallowed at once whether it be a living fly or an artificial one.

Sherrington in discussing this reaction divides it into an anticipatory phasefixation, coordination of somatic movement for the leap and seizure-and a consummatory reaction of mastication, swallowing, etc. It is the latter alone which gives satisfaction and in the interval which elapses between the beginning of the anticipatory reaction and the consummatory reaction we shall find the key to the problem of cortical function.

The whole feeding reaction in the lowest animals is so far as we can judge a blind reflex; the consummatory phase is largely so even in the highest animals, for once a morsel is in the mouth the processes of mastication and deglutition go on quite automatically. 
With the anticipatory phase, however, the case is quite different. The more complex the feeding act becomes, the more prolonged and difficult is this phase of the process. In the case of carnivorous vertebrates the prey must be recognized at a distance and carefully stalked and attacked from the most advantageous side, and all of these details will vary with each trial. No combination of simple reflex arcs can be laid down in advance within the nervous system which will be adequate to meet the infinite variations of these problems.

We may hypothecate the course of the evolution of this reaction as follows: In the lower animals, as in the spinal cords of the higher ones, the whole formatio reticularis, or correlation tissue, is relatively unspecialized and receives all kinds of afferent impulses from the primary sensory centers; these in turn it delivers over to the final common efferent pathways. There is thus a constant collateral avenue of nervous discharge through the reticular formation parallel with that in the primary reflex ares and reinforcing, inhibiting or otherwise modifying these primary simple reflexes.

The character of the efferent discharge from this reticular formation will depend upon the sources and strength of the afferent impulses, the fluctuating internal resistance of the chains of neurones of which it is composed and other variable factors, some of which, like the resistance at the neurone thresholds, may be modified, as already pointed out, by repetition of function (habit formation).

The suprasegmental correlation centers present essentially the same dynamic aspect, but with the afferent pathways more sharply defined and limited and the whole more perfectly adapted to effect definite types of more complex correlation. Thus, the thalamus becomes a great center for the correlation of somatic reflexes and the hypothalamus for visceral and olfactory reflexes. Accordingly, all of the lower primary correlation centers send strong secondary tracts upward into the diencephalon.

Now to return to the feeding activities, so far as these are contact reactions, such as nosing about in the mud for food, the interval between the anticipatory and consummatory phases is not necessarily long and a very simple reflex mechanism is adequate to distinguish between food and other objects.

But in the more complex cases the interval between the anticipatory and consummatory phases is occupied by the discharge into the higher correlation centers of a series of momentarily changing stimuli from the distance receptors, and the later acts of this phase will be the resultants of all of these influences plus the effects in the centers themselves of vestiges of similar reactions in the past. The whole system is in a state of neural tension which varies constantly as new impulses from the periphery reverberate through its substance. The high neural resistance of this complex tissue raises the threshold of discharge from it so that a certain summation of stimuli takes place before the tension is relieved by a discharge of the neural energy into the lower mechanism of the consummatory reaction, which is already so adjusted as to perform its functions when once actuated more or less mechanically and therefore without the development of such internal resistance as characterizes the anticipatory mechanism.

In the storm and stress of this interval just preceding the consummatory reaction the higher mental faculties are born.

The stream of nervous influences pouring into the higher correlation centers from 
the peripheral sense organs contains many elements of no significance to the immediate capture of the quarry. These stimuli the animal learns to ignore, perhaps in the first instance unconsciously, by an application of the biological law of habit; for those reflex ares which have adaptive value in this particular situation will lead at once to the desired consummatory reaction, leave their permanent vestiges in the nervous system and so be more easily repeated, while the irrelevant stimuli do not lead to a relief of the tension, come to nothing and leave no such vestiges. Upon later repetition of the series, the adaptive stimuli find a more open path through the nervous system than the non-adaptive, and accordingly they from the start tend to set the direction of the nervous discharge through the correlation centers, and during this process the sense organs are reflexly adjusted to the sources of these relevant stimuli to the exclusion of the irrelevant. This is the origin of attention.

The analysis of other types of distance reactions, such as avoidance of enemies, search for mates, etc., would show for them a similar significance for psychogenesis. The important point is that these complex forms of distance reaction demand for their highest efficiency greater flexibility and modifiability of response than do the visceral and contact reactions. Here only is a high degree of intelligence necessary. The cortex cerebri dominates cerebral architecture only in mammals where complex anticipatory reactions dominate the behavior, and foresight, literal and figurative, plays the leading rôle.

The cerebral cortex is a correlation center of a higher order, $i$. e., farther removed from the primary sensori-motor reflex ares, than those of the brain stem. It is not different in kind from those centers, but only in the extent of its removal physiologically from the primary centers and the nature and complexity of the associational connections within it. In the lower vertebrates the steps by which it has been gradually lifted above the lower correlation centers can now be traced with a considerable degree of precision. Some of this evidence will be reviewed in the symposium on comparative neurology to be held to-morrow in the meeting of the Association of Anatomists. We have time here merely to state in brief summary a few salient features.

We owe to the genius of Edinger the suggestion that the earliest stages in the origin of the peculiarities of the cerebral hemispheres must be sought in a study of the character of the reflexes connected with the nose and lips, particularly the feeding reactions. These have been termed collectively the "oral sense" (Edinger) or "Schnüffelsinn" (Kappers) and may perhaps best be called the muzzle reflexes.

In lower vertebrates the sense organs of the nose are probably the most important receptors in the muzzle reflex complex, and these are distance receptors and not contact receptors. Accordingly, the cerebral hemispheres were built up on the basis of the olfactory correlation centers, or rhinencephalon. In fishes, long before we find a true cerebral cortex, ascending tracts pass from the visceral sensory centers of the hypothalamus (probably mainly gustatory in function) and from the somatic centers of the thalamus and midbrain (mainly tactile in function) to enter the large forebrain correlation centers related to the olfactory apparatus. The association of these sensory elements and their return motor tracts produces the socalled corpus striatum of fishes, an apparatus which is probably largely concerned with reflexes of the nose, lips and mouth.

In Amphibia important optic projection 
fibers are added, passing from the external geniculate body of the thalamus to the hemispheres, and also acoustic fibers from the inferior colliculus of the midbrain. Though there is no true cerebral cortex here, the tissue from which it is to arise in reptiles can be definitely identified and this tissue is in the frog clearly divided into a medial part, serving primarily the correlation of olfactory and visceral reflexes, and a lateral part, serving primarily the correlation of olfactory and somatic reflexes. The former gives rise in higher animals to the hippocampus, the latter to the pyriform lobe (uncus), while the rest of the cortex, or neopallium, is in these animals differentiated dorsally between these two masses and serves chiefly for the correlation of non-olfactory reactions.

The two parts of the pallium which we call archipallium and neopallium (i. e., olfactory and non-olfactory cortex) are not of different age, as the names imply. They probably both arose at the same time to serve the delicate discriminative reactions of the muzzle reflexes. Their precursors are found in fishes and amphibians, where their cells are mingled in an undifferentiated tissue which has been called by some authors the epistriatum. They finally (in reptiles) become separated and within each division in mammals subordinate "areas" with more or less characteristic connections are differentiated. The incompleteness of this differentiation is responsible for much of the controversy which has waged regarding the presence and significance of localizable cortical areas.

No cortical area can properly be described as the exclusive center of a particular function. In higher mammals it is true that the several final common paths for particular effectors leave more or less clearly defined areas of cortex and that the several kinds of sensory projection fibers terminate in other more or less definite areas. But these so-called sensory and motor areas are in no proper sense centers for the performance of definite functions. Such a "center" is merely a nodal point in an exceedingly complex system of cells and fibers which must act as a whole in order to perform any function whatsoever. Their relation to cerebral functions is analogous to that of the railway stations of a big city to traffic, each drawing from the whole city its appropriate share of passengers and freight, and their great clinical value grows out of just this segregation of fibers of like functional systems in a narrow space, and not to any mysterious power of generating psychic or any other special forces of their own.

The essence of cortical function is correlation and a cortical center for the performance of a particular function is a physiological absurdity, save in the restricted sense described above, as a nodal point in a very complex system of associated conducting paths. Those reflexes whose simple functions can be localized in a single center have their mechanism abundantly provided for in the brain stem.

In the broad view we may say that intelligence is a function of the cerebral costex, but only in the sense that here are found the most complex correlations in the chain of vital response whose initial phase is to be sought in the environment which supplies the stimulus and whose final phase is also found in the changes wrought in the environment by the bodily reaction. A similar function is performed in a less perfect way in lower animals which lack the cerebral cortex, and doubtless even in man the subcortical nervous apparatus still plays an important part in all conscious processes. 
The resting brain is probably normally during life in a state of neural tension in more or less stable equilibrium. An effective stimulus disturbs this equilibrium and the precise effect will depend upon variable synaptic resistance or neurone thresholds which change with different functional states of the organism as a whole and of the brain in particular. If this activity involves the cerebral cortex of a human brain, it may be a conscious activity, the kind of consciousness depending on the kind of discharge. But the consciousness must not be thought of as localized in any cortical area.

The discharge in question may reverberate to the extreme limits of the nervous system and the peripheral activities may be as essential in determining the conscious content as the cortical. Indeed, we have considerable evidence that many of our conscious acts take their most distinctive psychic qualities from the "back stroke," or reverberation of a neural discharge from the periphery back to the cortex.

Thus far we have tacitly assumed that consciousness is an integral part of the complex of bodily functions. This assumption lies at the basis of most modern work in the field of comparative psychology and rests on the thoroughly scientific basis of a large body of observation and inference. In the nature of the case demonstrative proof is impossible, for consciousness as $I$ know it is a purely individual experience; but without the assumption that like behavior in other men implies experience like my own in similar circumstances the science of psychology can not go on, and without the further assumption that other animals have like experience in proportion as their behavior is like my own comparative psychology is an impossible science.

Now keeping in mind the dynamic conception of the workings of the nervous mechanism developed above, let us see whether the introspective examination of some very simple conscious reaction can be put into scientific relation with other biological processes, or whether it must be left out of our science in the cold isolation of mere epiphenomena and similar metaphysical abstractions.

An unfamiliar or unexpected sensation is experienced; let us say a noise. There is a moment of hesitancy while the sensory stimuli, numerous awakened memory vestiges, each perhaps with its own emotional coloring, and many half-formed impulses, surge in consciousness. When the problem presented by the new situation is solved, the mental tension is relieved and the intellectual process crystallizes at once into action. I am thinking about it no longer: I have got an idea; and the appropriate act follows immediately and automatically unless inhibited by some extraneous influence. Here we have an active and complex interplay of conscious elements corresponding to what in the objective manifestation we called the anticipatory phase of the reaction, and the conscious process comes to an abrupt end as soon as it passes over into the already stereotyped form of reaction. That is, this conscious process ends, though of course it may be followed at once by another similar chain of events.

Here we see how intelligence and feeling are developed as the servants of action. They do not appear so long as the action can be effected without them and they vanish as soon as the reflex machinery of an adaptive action is set in motion. Consciousness is a functional phase of the more complex mechanism of those higher non-stereotyped actions for which the reflex machinery is inadequate, in much the same way that the tropisms of Paramecium and the sucking reflex of an in- 
fant are functional phases of the simple inborn neuro-muscular mechanisms of these organisms.

We do not know whether any glimmer of consciousness is involved in these simple processes; but if we study the behavior of the whole series of animals from $A m œ b a$ to man objectively, we can find no point where to an outside observer the behavior which we called discriminative reaction in a protozoan passes over into conscious choice as we see it in our fellow men. The series of stages is complete and unbroken until I begin to study my own choices, when I find by introspection that the whole mental fabric is involved-ratiocination, swayed perhaps now this way, now that, by waves of feeling, and finally will. Out of the psychic chaos of hesitation and doubt I say, and I say truly, "I have made up my mind," and action results.

Now this seems to me a very different thing from the discriminative reaction of an amœba, or even the deliberately judicious act of a fellow man. Both of the latter are alike in that $I$ do not directly experience feeling, will, etc., in connection with them. Possibly if I could be successively for a time an amœba, a sea anemone, a frog, a man and all the types of animals between by the act of some benevolent Buddha, and if I could carry my memory of each stage through all of the others, then perhaps the psychic series would appear at the end a simple and unbroken graded series, as the objective physiological series does to me now.

Meanwhile, without intending at this time to penetrate far into a field of philosophical speculation which clearly lies beyond the present limits of biological science, I wish to make one further observation on the great problem of the relation of mild and body. We have seen that animal bodies can be arranged in a graded series (not a simple linear series, to be sure, but a true series, nevertheless) of genetically related forms; that animal activities can be arranged in a similar graded series of functions; and that these two series are closely related. In fact, they are absolutely inseparable except by logical abstraction or some artificial scientific procedure, for their respective members are related to each other as structure and function, as objects and their properties, and neither can exist apart from the other.

There is, however, a third series, the psychic series, of which I know directly only one member-my own experience. But I have satisfactory indirect evidence that the psychic series also extends for at least a part of the distance parallel with the other two. And wherever I can analyze this evidence it teaches that psychic processes, like physiological processes, are related to living bodies as functions of their structures. If it be permissible to generalize from these facts, and say that both physiological and psychological processes may be included in the one category of function, we may conclude that we have not to reckon in science with three independent genetic series, anatomical, physiological and psychological, but with one-a single series of functioning structures, whose genetic continuity is unbroken from its simplest to its most complex members and which can be dissociated, as is commonly done, only by doing violence to truth.

The present isolated position of the three disciplines of anatomy, physiology and psychology is due partly to the exigencies of practical pedagogical and methodological convenience and partly to our incomplete knowledge of the facts.

It is perhaps well to add that the position here defined is as far removed as 
possible from that naïve materialism which would postulate a single series of objects as the ultimate realities with more or less adventitious functions pertaining to them as epiphenomena. The analysis here attempted is merely pragmatic and proximate, not ultimate, and it leaves quite to one side and untouched the metaphysical problem of the ultimate nature of the phenomenal series, whether it is materialistic or idealistic or both or neither.

Looking back now over the field which we have traversed, in our analysis of the behavior of animals and its mechanisms we start with the tropism and the reflex. This type of response is in some of its simpler phases indistinguishable from the reactions of dead machines to the forces which actuate them. But the more complex reflexes, on the other hand, grade over into those behavior types which we call intelligent. No one has yet succeeded in formulating a clear-cut definition of the limits of the reflex at either its lower or its higher extreme, and perhaps no one ever will; for the whole list of behavior types from machines to men probably forms a closely graded series.

Even the simpler reflexes exhibit a measurable refractory phase, or pause, in the center where the afferent impulse is made over into the efferent. When reflexes are compounded, there is another factor which may tend to modify or delay the response. This is the dilemma which arises when two or more reflex centers are so related that a given afferent impulse coming to one of them may take any one of several final common paths to the organs of response. The reflex response which actually emerges in such a case will generally be the adaptive one, $i$. $e$, the one which is best for the organism. The selection of the adaptive response in such a case may be termed physiological choice, and it always involves a lengthening of the refractory phase. ${ }^{5}$ In the neural tensions of the refractory phase of physiological choice we find the germs of the complex anticipatory reactions which in turn have nurtured the awakening intelligence.

I have atempted to illustrate the thesis that the comparative study of animal behavior in the broadest sense of the term is as essential as other branches of physiology to the comprehension of animal structures and that the enlargement of our knowledge of scientific fact in this field will contribute greatly to the more perfect integration of the three great branches of biology-anatomy, physiology and psychology-and the correlation of the whole with other departments of knowledge. Our philosophy of nature is sound just in proportion as we succeed in effecting these correlations of experience.

\section{Judson HerRick}

\section{THE ALASKAN FUR-SEALS}

WHEN, on January 1, 1909, the management of the Alaskan fur-seal fisheries was transferred to the United States Bureau of Fisheries, the Secretary of Commerce and Labor designated Dr. David Starr Jordan, Dr. Leonhard Stejneger, Dr. C. Hart Merriam, Dr. Frederic A. Lucas, Dr. Chas. H. Townsend, Hon. Frank H. Hitcheock and Hon. Edwin W. Sims, to act as an advisory board to recommend measures designated to conserve this valuable animal life now being exterminated through sea-killing of breeders. On November 23, last, this board met at the Bureau of Fisheries in Washington and adopted the following recommendations, which

${ }^{5}$ Physiological choice, in fact, is not dependent upon a nervous system at all, but has been demonstrated in rudimentary form even among Protozoa, though it remains on a very low plane in these organisms.

${ }^{1}$ Published by permission of Hon. Geo. M. Bowers, U. S. Commissioner of Fisheries. 